\title{
Research on the Water Resources Carrying Capacity in Beijing Based on the System Dynamics Model
}

\author{
Yang Bai \\ School of North China Electric Power University, Baoding 071000, China. \\ 838264478@qq.com
}

Keywords: System dynamics model; dynamic simulation; water scarcity.

\begin{abstract}
According to water scarcity, using the system dynamics (SD) theory as a guide, this report makes some quantitative and qualitative analyses on the trend of water resources carrying capacity (WRCC) in Beijing. According to several corresponding reasons, counter-measures are proposed to diminish the degree of water scarcity. Comparing with the former result, the later result by using the SD model shows that the intervention plan has a positive effects on water shortage.
\end{abstract}

\section{Introduction}

Firstly, the report gives the definition of water resources carrying capability, i.e. the ratio of water supply and water demand. Then we are to establish a model to measure this capacity from the aspects of supplying and consuming. Since the factors in water resources system are complex and dynamic, the System Dynamics model is adopted to the problem [1]. The water resources system is divided into six subsystems which are interrelated to each other. The relationships of all the factors in the subsystems are to be analyzed to establish kinetic equation to quantify the issue.

Secondly, according to the latest data on Beijing water resources, the WRCC graph is drawn by using Vensim to quantify the current situation. The result shows that Beijing will be short of water supply in the year 2027.

Last but not least, several measures can be taken out. Possible effects of the suggested policies on the region are quantified and examined by using Vensim software. The capacity trend in Beijing with the policy intervention from 2013 to 2060 shows that water scarcity in Beijing is still unavoidable, but water supply shortage will be deferred until the year 2048.

\section{Model of WRCC Based on the SD Model}

\subsection{Definition of WRCC.}

To represent the ability of a region to provide water availability to meet the needs of the population, we define water resources carrying capacity as the ratio of total water supply to total water demand.

$$
W R C C=W_{p} / W_{u}
$$

\subsection{System Analysis.}

This report aims to divide the water resources system in the chosen region into six subsystems, including population system, industrial system, agricultural system, economic system and water resources system, to analyze the causal relations causing water scarcity. The water resources system mainly contains return water, underground water and surface water, so it is these three resources that need be taken into consideration. Similarly, in the agriculture system, it is the agricultural acreage, the water-saving technology and irrigation water that should be primarily taken into account. Factors to be considered in other subsystems and the corresponding relationship among those factors will be demonstrated in the following causal loop diagram. 


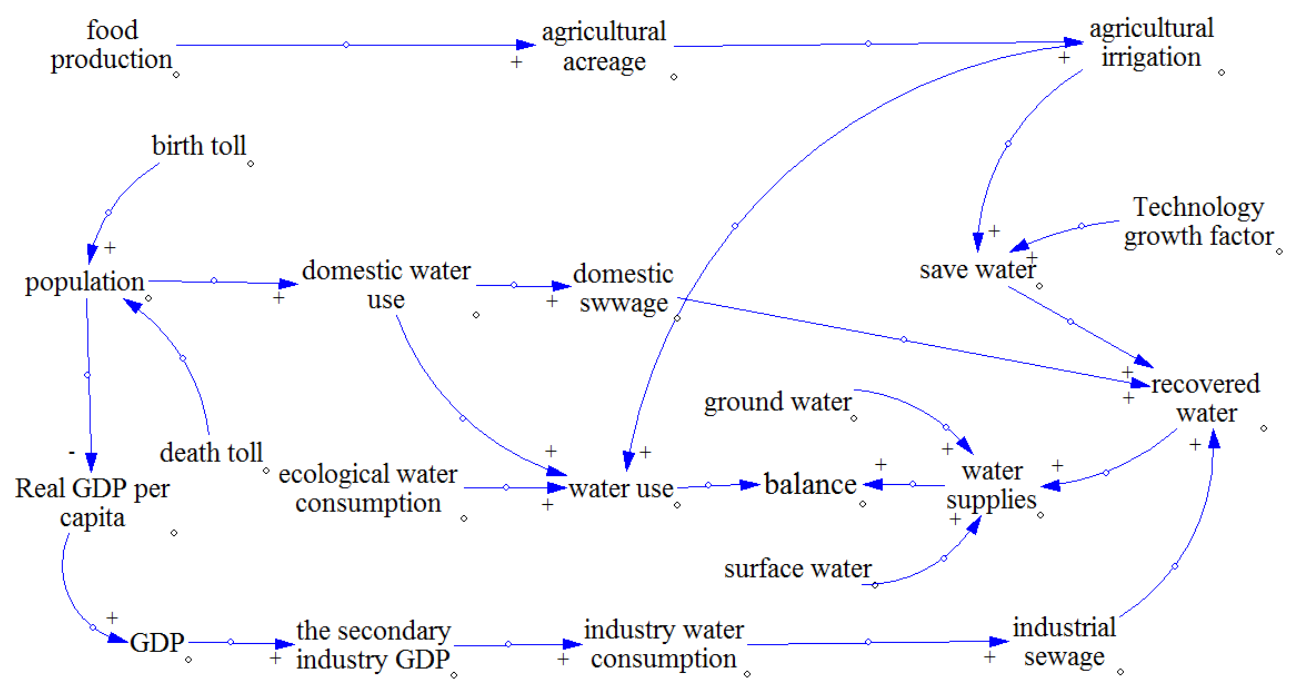

\subsection{Structural Equation.}

Fig. 1 Causal loop diagram

We are to settle a set of structural equations to plant into our model to get the calculated results. Generally, the form of the equation of state is shown below:

$d L / d t=f\left(X_{i}, R_{i}, A_{i}, P_{i}\right)=R$

Where $\mathrm{X}$ is the object variable symbol, $\mathrm{R}$ is rate equation, $\mathrm{A}$ is instrumental variable, $\mathrm{P}$ is parameter, $\mathrm{L}$ is equation of state variable.

Differencing format stated below:

$X(t+\Delta t)=X(t)+f\left(X_{i}, R_{i}, A_{i}, P_{i}\right) \times \Delta t$

All the equations of each subsystem needed is listed in the following pages [2].

The equations of population subsystem based on the SD model.

$\left\{\begin{array}{l}P(t)=P(t-1)+B(t-1)-D(t-1) \\ B(t-1)=P(t) \times B_{r} \\ D(t-1)=P(t) \times D_{r}\end{array}\right.$

The equations of industrial subsystem based on the SD model.

$\left\{\begin{array}{l}I_{m}(t)=I_{m}(t-1)+\Delta I_{m i}(t-1)-\Delta I_{m d}(t-1) \\ \Delta I_{m i}(t-1)=I_{m}(t-1) \times \Delta I_{m i r} \\ \Delta I_{m d}(t-1)=I_{m}(t-1) \times \Delta I_{m d r}\end{array}\right.$

The equations of agricultural subsystem based on the SD model.

$\left\{\begin{array}{l}A(t)=A(t-1)+\Delta A_{i}(t-1)-\Delta A_{d}(t-1) \\ \Delta A_{i}(t-1)=A(t-1) \times L_{r} \\ \Delta A_{d}(t-1)=A(t-1) \times C_{r}\end{array}\right.$

The equations of economic subsystem based on the SD model.

$G(t)=G(t-1)+\Delta G$

The equations of water subsystem based on the SD model.

$$
\left\{\begin{array}{l}
W_{B}=W_{p}-W_{u} \\
W_{p}=W_{g}+W_{s}+W_{b} \\
W_{b}=W_{d s}+W_{i s}+W_{s} \\
W_{u}=W_{d}+W_{i}+W_{a}+W_{e}
\end{array}\right.
$$

What to be remarked is that we see all the rate in this model as constants for simplifing the problem. With all the equations established, we can plant them into the flow chart of the system model by using Vensim. With all the initial values needed given, the results of the model can be attained. 
Since the model can show the trends of total water supply and total water use, the prediction in 15 years can be obtained by planting the initial data into Model one. The whole data needed is the data of 2013, which is the newest data we can find from the website [3]. Therefore, with given data planting into the model, the curve of water-supplying capacity is shown in the curve below:

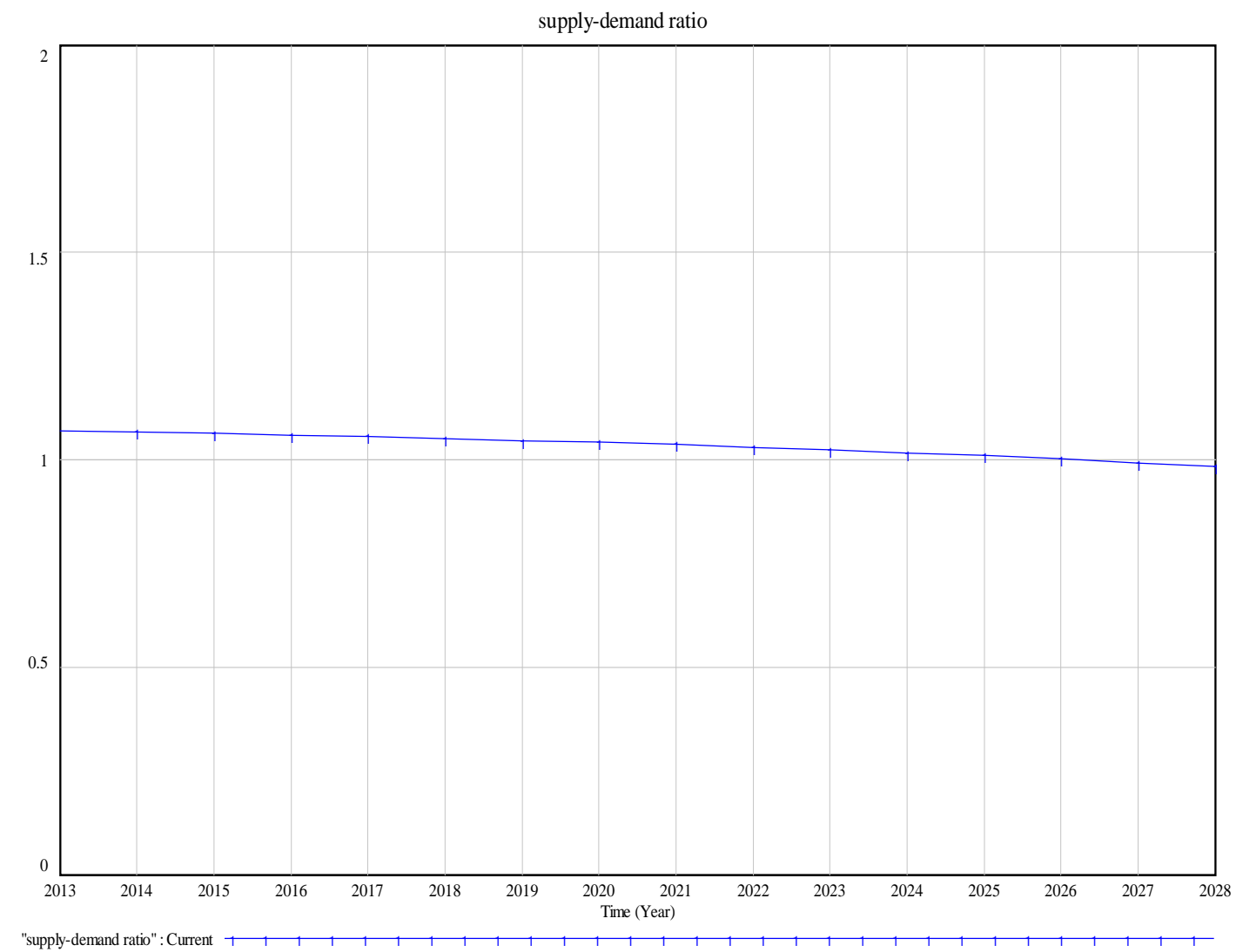

Fig. 2 Results by using Vensim

From the picture above, we can reach a conclusion that water supply will always be slightly larger than the amount of water used before 2027, that is, the appearance of insufficient water supply will not occur until 2027. Starting from the time 2016, there will appear water scarcity after eleven years in Beijing. The value of water supply capacity defined early tends to be lower than one, suggesting that without measures taken, the water supply capacity of BeiJing will gradually diminish, and then aggravate the water shortage.

\section{Advanced Model with Intervention}

Since the former model is established without intervention, if to predict the trend of water supply capacity, the model need to be improved. The intervention policy need to be quantified as a constraint to apply to the original model, through which we can analyze the effect of intervention policy on easing the water pressure [4,5].

The quantification for water diversion:

$$
\left\{\begin{array}{l}
W_{p}^{\prime}=W_{g}+W_{s}^{\prime}+W_{b}+W_{t} \\
\left.W_{t}=\text { random } \quad \text { uniform[5exp(8), } 8 \exp (8), 1\right] \\
W_{s}^{\prime}=W_{s}+W_{c} \\
\left.W_{c}=\text { random uniform[8exp(6), } \exp (7), 1\right]
\end{array}\right.
$$

Where $W_{p}^{\prime}$ is total water supply under intervention plan, $W_{s}^{\prime}$ is total surface water under intervention plan, $W_{t}$ is total diverted water.

The quantification for climate change: 
$\left\{\begin{array}{l}W_{s}^{\prime}=W_{s}+w_{c} \\ w_{c}=\text { random } \quad \text { uniform }[8 \exp (6), \exp (7), 1]\end{array}\right.$

Where $W_{s}^{\prime}$ is total surface water under intervention plan, $w_{c}$ is the increasing rainfall under intervention.

The quantification for policy on water use:

$\left\{\begin{array}{l}S_{i r}=\left(T_{g r}+1\right) \times 0.05 \\ T_{g r}=0.001 \times \exp (0.01 t) \\ S_{a}=\left(T_{g r}+1\right) \times 0.05\end{array}\right.$

Where $T_{g r}$ is technical coefficient, $S_{i r}$ is the rate of industrial sewage treatment, $S_{a}$ is the rate of agricultural saving water.

The quantification for technology improvement:

$\left\{\begin{array}{l}W_{d}^{\prime}=W_{d}-5 p_{d} \\ p_{d}=\text { random uniform }[0,20,1] \\ W_{i}^{\prime}=W_{i}-\exp (-6) p_{i}-T_{g r} \times \exp (-6) \\ p_{i}=\text { random uniform }[10,30,1]\end{array}\right.$

Where $W_{d}^{\prime}$ is total domestic water use under intervention plan, $W_{i}^{\prime}$ is total industrial water use under intervention plan, $p_{d}$ and $p_{i}$ is the quantification of domestic and industrial water price changes.

With the new established equations planted into the model, results are shown in the Fig.3. We can reach a conclusion that the designed intervention plan has an obvious effect on alleviating the press of water scarcity. Water scarcity will not appear until 2048, suggesting water will become a critical issue in the future. This leaves us an urgent task to solve.

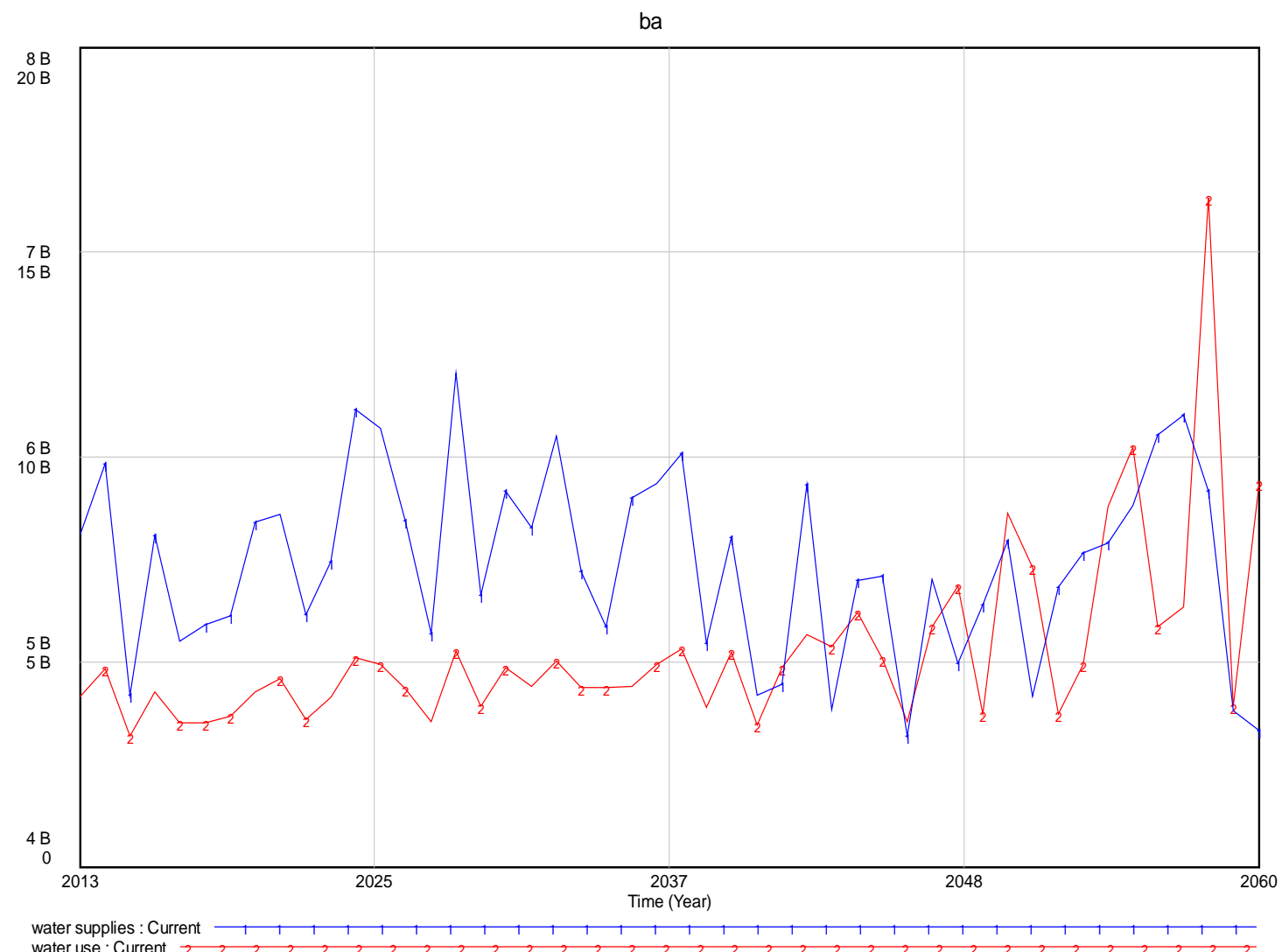




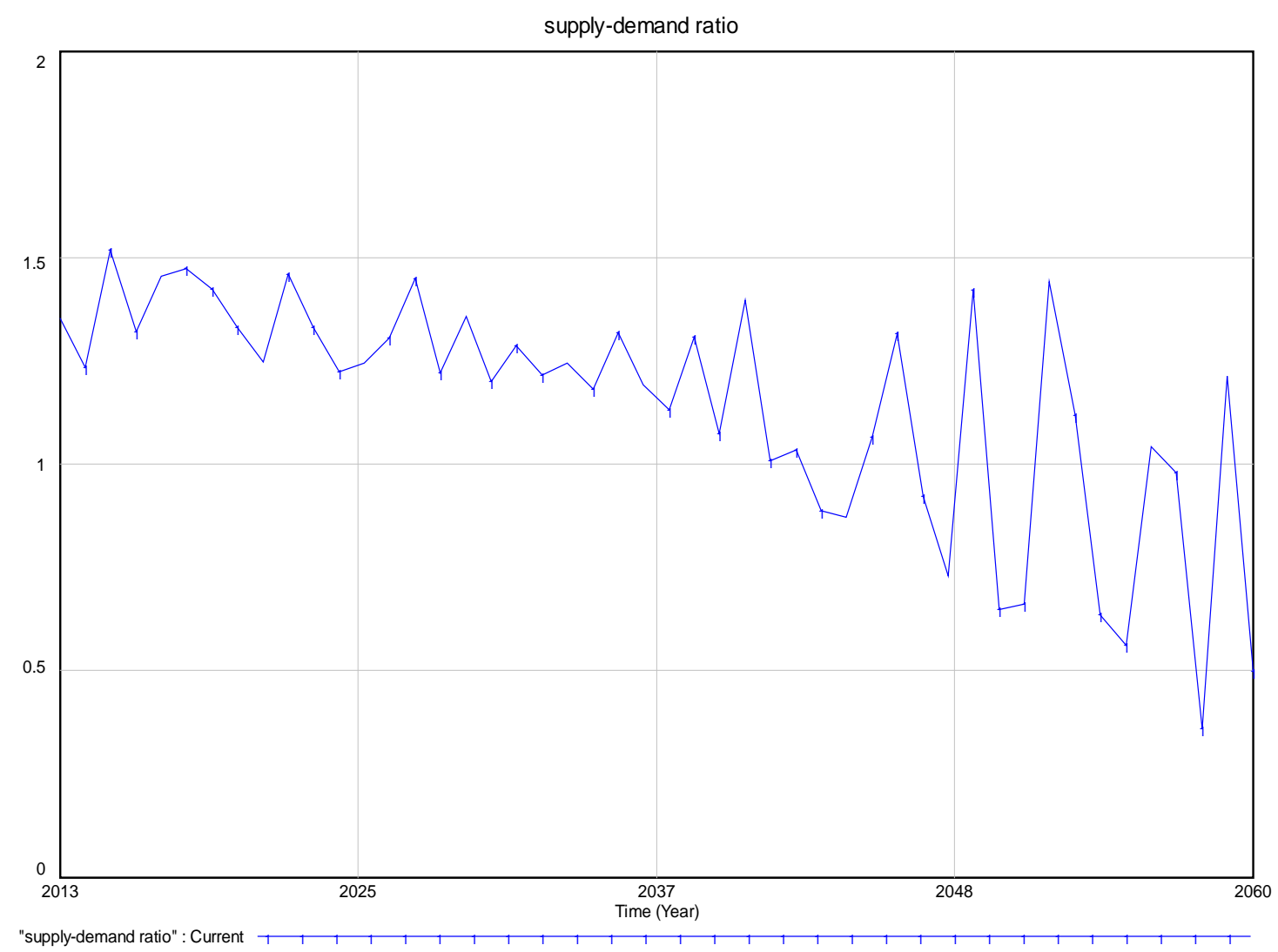

Fig. 3 Results of water supply capacity under intervention

\section{Summary}

With WRCC defined, water supply capacity of Beijing is estimated by establishing the SD model, showing a negative water supply trend. Therefore, intervention plan should be taken out immediately. With designed scheme quantified and improved model, the results acquainted by Vensim software shows that the designed intervention plan has a positive effect on easing scarcity pressure. But the water scarcity will still become an issue in the future, requiring more attention of government.

\section{References}

[1] Ines Winz, Gary Brierley. The Use of System Dynamics Simulation[R]. Water Resour Manage , 2009.

[2] Qiong A. Study of Water Resources Carrying Capacity Based on System Dynamics Model in Tianjin City [D]. Tianjin University, 2008.(China)

[3] Information on: http://www.bjstats.gov.cn/

[4] Chen CM, Zhang CY, Ma CX.1999.System Dynamics for Zhengzhou Land Resource Bearing Capacity. Journal of Hehai University27 (1):53-56[in Chinese].

[5] Xu ZQ, Huang TL. 2005. Optimization of the Decentralized Sanitation and Reuse System. Journal of Xi'an University of Arch. \& Tech. (Natural Science Edition) 37(2):164-168[in Chinese]. 